\title{
TRAPPING GAMES ON RANDOM BOARDS
}

\author{
BY RIDDHIPRATIM BASU ${ }^{*}, 1$, ALEXANDER E. HOLROYD ${ }^{\dagger}$, \\ JAMES B. MARTIN ${ }^{\ddagger}, 2$ AND JOHAN WÄSTLUND ${ }^{\S, 3}$ \\ Stanford University*, Microsoft Research ${ }^{\dagger}$, University of Oxford ${ }^{\ddagger}$ \\ and Chalmers University of Technology $y^{\S}$
}

We consider the following two-player game on a graph. A token is located at a vertex, and the players take turns to move it along an edge to a vertex that has not been visited before. A player who cannot move loses. We analyze outcomes with optimal play on percolation clusters of Euclidean lattices.

On $\mathbb{Z}^{2}$ with two different percolation parameters for odd and even sites, we prove that the game has no draws provided closed sites of one parity are sufficiently rare compared with those of the other parity (thus favoring one player). We prove this also for certain $d$-dimensional lattices with $d \geq 3$. It is an open question whether draws can occur when the two parameters are equal.

On a finite ball of $\mathbb{Z}^{2}$, with only odd sites closed but with the external boundary consisting of even sites, we identify up to logarithmic factors a critical window for the trade-off between the size of the ball and the percolation parameter. Outside this window, one or the other player has a decisive advantage.

Our analysis of the game is intimately tied to the effect of boundary conditions on maximum-cardinality matchings.

1. Introduction. Consider the following natural two-player game on an undirected graph. A token is located at a vertex, and the players take turns to move. A move consists of moving the token along an incident edge to a new vertex that has never been visited before by the token. If a player has no possible move, she loses (and the other player wins). We call this game Trap (since the goal is to trap one's opponent).

We are concerned with optimal play. Thus, a strategy for the first or second player is a map that assigns a legal next move (where one exists) to each position. (A position comprises a location of the token and a set of visited vertices.) Given

Received May 2015; revised February 2016.

${ }^{1}$ Supported by a University of California, Berkeley Graduate Fellowship.

${ }^{2}$ Supported by EPSRC Fellowship EP/E060730/1.

${ }^{3}$ Supported by the Swedish Research Council, the Knut and Alice Wallenberg Foundation and the Göran Gustafsson Foundation.

MSC2010 subject classifications. 05C57, 60K35, 05C70.

Key words and phrases. Combinatorial game, percolation, maximum matching, maximum independent set, boundary conditions. 
a graph and an initial vertex (at which the token starts), we say that the game is a win for the first or second player respectively if that player has a winning strategy, that is, a strategy that results in a win, no matter what strategy the other player uses. If the graph is finite, it is easy to check that the game is a win for exactly one player. In an infinite graph, it is possible that neither player has a winning strategy, in which case we say that the game is a draw (with the interpretation that the game continues forever with optimal play).

As we shall see, the outcome of Trap is intimately tied to the properties of maximum-cardinality matchings, and draws relate to sensitivity of such matchings to boundary conditions. We shall consider another related game, which we call Vicious Trap, in which a player, after making a move, is allowed to destroy (i.e., delete from the graph) any subset of the vertices that he could have just moved to; in this case, the outcome is related to maximal independent sets.

Trap and similar games have been studied on finite graphs from the point of view of computational complexity. The link to matchings was given in [7] (see also [3], Exercise 5.1.4), where it was used to observe that the outcome of the game can be computed in polynomial time. In contrast, related games where the edges are directed, and/or where one is allowed to visit the same vertex twice but not to use the same edge twice, are shown in $[7,15,17]$ to be PSPACE-complete. In [16], misère versions of these games (where a player unable to move wins) are also shown to be PSPACE-complete. (In these contexts, Trap has been called Undirected Vertex Geography, but here we prefer the shorter and more evocative name. The name Slither has also been used, for example, in [6], although it had already been used for a slightly different game in [2,9].)

In this article, we focus on infinite graphs, and the resulting possibility of draws, especially in a random setting — so far as we know, these directions are novel. (See $[12,13]$ for research involving some of the current authors of similar questions in directed graphs.) In a related direction, in [20], results about minimum weight matchings in random edge-weighted graphs were derived from analysis of a related game called Exploration. Two-player games also arise as a tool in first-order logic, including in probabilistic settings-see [19].

We are interested in playing Trap on a percolation cluster. Let $G$ be an infinite connected graph, let $p \in[0,1]$ and let each vertex of $G$ be declared closed with probability $p$, and otherwise open, independently for different vertices. Consider Trap on the subgraph of $G$ induced by the set of open vertices. (Equivalently, we play on $G$ but with moves to closed vertices forbidden. For convenience, the game is declared a first player win if the initial vertex is closed.) We emphasize that the random subgraph is assumed known to both players when deciding on their strategies. If $p$ exceeds one minus the critical probability for site percolation on $G$, then almost surely the subgraph has no infinite components and, therefore, the game cannot be a draw. For many graphs (including the hypercubic lattice $\mathbb{Z}^{d}$ ), the latter conclusion can be extended to a strictly longer interval of $p$ using the method of essential enhancements [1]. On $\mathbb{Z}^{d}$ itself (i.e., on the open subgraph 
TABLE 1

Conventions for bipartite graphs

\begin{tabular}{lll}
\hline $\begin{array}{l}\text { Odin moves to } \\
\text { Eve moves to }\end{array}$ & $\begin{array}{l}\text { odd vertices, } \\
\text { even vertices, }\end{array}$ & which are closed with probability $p$. \\
which are closed with probability $q$.
\end{tabular}

with $p=0$ ), Trap is easily seen to be a draw. The regime of small positive $p$ seems to be the most interesting.

A very interesting and challenging open question is whether there exists $p>0$ for which Trap on the open subgraph of $\mathbb{Z}^{d}$ (started from the origin) is a draw with positive probability. As we discuss later, simulations lend some support to a negative answer in dimension $d=2$. On the other hand, draws $d o$ occur on certain random trees [13], while variants of the model on directed lattices exhibit draws in dimensions $d \geq 3$ but not in $d=2$ [12].

Suppose now that the graph $G$ is bipartite, and call vertices in its two classes odd and even. A natural extension of the above model is to declare odd and even vertices closed with respective probabilities $p$ and $q$ (with different vertices still receiving independent assignments). Given an initial vertex (which may be odd or even), one player always moves from even vertices to odd vertices. Call this player Odin, and the other player Eve. We summarize these conventions in Table 1. The probability that Odin wins is nonincreasing in $p$ and nondecreasing in $q$, and viceversa for Eve. (Indeed, introducing more closed odd vertices preserves all winning strategies for Eve but cannot create winning strategies for Odin.) However, there is apparently no obvious monotonicity argument for the probability of a draw.

If $p>q$, then we should expect Eve to have an advantage. Our first main result states that this advantage is decisive in the extreme case $q=0$. We prove this in all dimensions $d$, but on a slightly nonstandard lattice when $d \geq 3$. The bodycentered hypercubic lattice is the graph $\mathbb{B}^{d}$ whose vertices are all elements of $\mathbb{Z}^{d}$ having coordinates that are all even or all odd (called even and odd vertices, resp.), and with an edge between vertices $u$ and $v$ whenever $\|u-v\|_{\infty}=1$. Note that $\mathbb{B}^{2}$ is isomorphic to the usual square lattice $\mathbb{Z}^{2}$. See Figure 1.
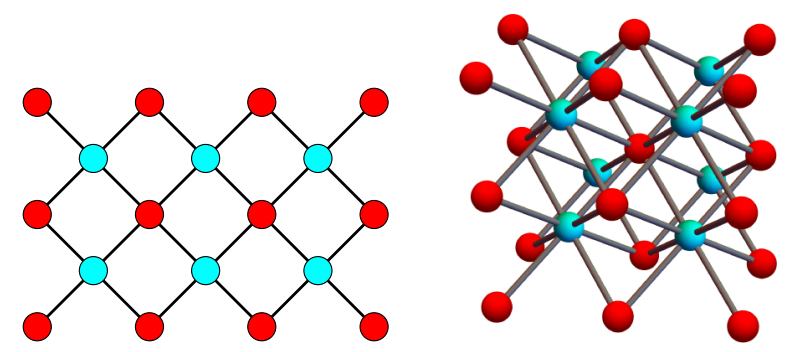

FIG. 1. The body-centered lattice in dimensions 2 and 3 (with colors indicating even and odd vertices). 
THEOREM 1. Let $d \geq 2$ and consider the body-centered lattice $\mathbb{B}^{d}$, with odd and even vertices closed with respective probabilities $p$ and $q$. Consider a game of Trap between Eve and Odin on the open subgraph of $\mathbb{B}^{d}$.

(i) Let $p>0$ and $q=0$. Almost surely, for every initial vertex, Eve wins.

(ii) For all $p>0$ there exists $q(p, d)>0$ such that if $q<q(p, d)$ then almost surely, for every initial vertex, the game is not a draw.

The key step in our proof of Theorem 1 will be to show the existence of finite regions with the property that if Odin enters one, he cannot escape. This is proved using a result of [18] on (modified) bootstrap percolation. To ensure that with high probability a region has the required properties for this argument, it must be very large, of order $\exp ^{d-1}(\lambda / p)$ for small $p$ (where $\lambda=\pi^{2} / 6$, and the exponential function is iterated $d-1$ times). This follows from results of $[4,5,10,11]$. The resulting lower bound on $q(p, d)$ is therefore very small: $O\left(1 / \exp ^{d-1}(\lambda / p)\right)$ as $p \rightarrow 0$. As we shall see, this bound is in fact very far from optimal, at least in dimension $d=2$.

It is likely that our methods could be adapted to prove that the conclusions of Theorem 1 hold also for the standard hypercubic lattice $\mathbb{Z}^{d}$ in all dimensions. Checking this entails adapting standard results of [18] to a variant of bootstrap percolation in a different combinatorial setting. We expect that such a proof would be rather messy (much more so than the proof in [18]) and perhaps very specific to $\mathbb{Z}^{d}$, although it is unlikely to involve fundamental new difficulties. (In particular, it appears to require separate induction steps for odd and even dimensions.)

Rather than pursuing this, we focus next on the more interesting question of obtaining tighter bounds involving finite regions in dimension 2 . This will yield improved bounds on $q(p, 2)$. Moreover, understanding the game on finite regions is an important step toward the main open question about the case $p=q$ as discussed earlier.

We restrict attention to the square lattice $\mathbb{Z}^{2}$ (i.e., the graph with vertex set $\mathbb{Z}^{2}$, and an edge between $u$ and $v$ whenever $\|u-v\|_{1}=1$ ). A vertex is called odd or even according to whether the sum of its coordinates is odd or even. We take $p>0$ and $q=0$ (so that only odd vertices can be closed), but we offset Eve's advantage by restricting to a finite region in a way that favors Odin. Specifically, let $n>0$ be an integer, and let $D_{n}$ be the subgraph of $\mathbb{Z}^{2}$ induced by the region $\left\{u \in \mathbb{Z}^{2}:\|u\|_{1}<2 n\right\}$. We call $D_{n}$ a diamond. Note that all internal boundary vertices of $D_{n}$ (i.e., those $u$ with $\|u\|_{1}=2 n-1$ ) are odd. We consider the game on the open subgraph of $D_{n}$. Thus, Odin is forbidden from moving to closed vertices, but Eve is forbidden from moving out of $D_{n}$. Intuitively, Eve tries to trap Odin using the closed odd vertices, while Odin tries to trap Eve against the boundary. (Equivalently, we can consider the game on the open vertices of $\mathbb{Z}^{2}$, but declaring a win for Odin if the token ever leaves $D_{n}$, or alternatively we can declare all vertices outside $D_{n}$ to be closed.) The progress of this game in a few cases is illustrated in Figure 2. 


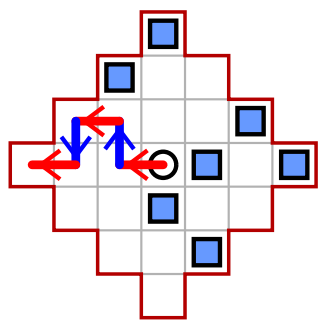

Odin starts and wins.

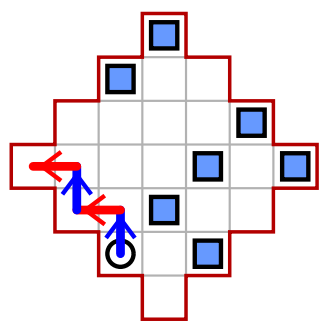

Eve starts and loses.

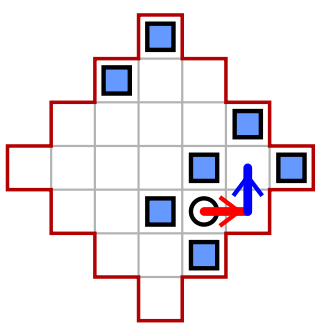

Odin starts and loses.

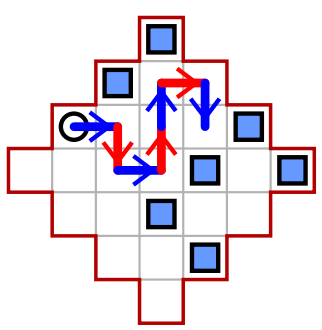

Eve starts and wins.

FIG. 2. Examples of optimal play on the diamond $D_{2}$, from four different initial vertices. Squares represent vertices, with closed (odd) vertices shown as filled squares, and the initial vertex marked with a circle. Odin's moves are red; Eve's moves are blue. (The colors of the boundary and the closed vertices reflect the fact that they are helpful to Odin and Eve, resp.)

It is easy to see that the probability that Eve wins (starting from the origin, say) is nondecreasing in $p$ and $n$. We address how these two effects compare with each other as $(n, p) \rightarrow(\infty, 0)$. We show that up to logarithmic factors, the critical regime is at $n=$ constant $/ p$; outside this window, one player has a decisive advantage, for essentially every initial vertex. In the following, "with high probability" means with probability tending to 1 .

THEOREM 2. Let odd and even vertices of the diamond $D_{n}$ be closed with respective probabilities $p>0$ and $q=0$, and consider a game of Trap. For every constant $c>0$, if $n<c /\left(p \log p^{-1}\right)$, then, with high probability as $p \rightarrow 0$, from every initial vertex in $D_{n}$, Odin wins.

The next result gives complementary conditions under which Eve wins, but now there are exceptional initial vertices near the boundary. Define $K_{0}=\left\{(x, y) \in \mathbb{Z}^{2}\right.$ : $|y|<x\}$, and for $k=1,2,3$ let $K_{k}$ be obtained by rotating $K_{0}$ counterclockwise by $\pi k / 2$ about the origin. We say that a vertex $u \in D_{n}$ is protected if each of the cones $u+K_{0}, \ldots, u+K_{3}$ contains some closed vertex of $D_{n}$. If the initial vertex is even and unprotected, then a simple winning strategy for Odin is to choose such a cone containing no closed vertex, and always move in the direction of that cone (e.g., rightward in the case of $u+K_{0}$ ). 
THEOREM 3. Let odd and even vertices of the diamond $D_{n}$ be closed with respective probabilities $p>0$ and $q=0$, and consider a game of Trap. There exists a constant $C>0$ such that, if $n>\left(C \log p^{-1}\right) / p$, then, with high probability as $p \rightarrow 0$, from every odd vertex and every protected even vertex of $D_{n}$, Eve wins.

It is straightforward to check that for a fixed constant $C^{\prime}$, with high probability, every even vertex in the set

$$
S:=\left\{(x, y) \in D_{n}:\left(2 n-\left|\frac{x+y}{2}\right|\right)\left(2 n-\left|\frac{x-y}{2}\right|\right)>\frac{C^{\prime} \log p^{-1}}{p}\right\}
$$

is protected. Since $\left|D_{n} \backslash S\right|=O\left(p^{-1} \log ^{2} p^{-1}\right)$ as $p \rightarrow 0$ (uniformly in $n$ ), in the situation of Theorem 3 , the conclusion of the theorem applies to all but a fraction $O(p)$ of the even vertices of $D_{n}$ with high probability as $p \rightarrow 0$. (We justify these remarks in Section 6.)

One consequence of Theorem 3 is that for the 2 -dimensional lattice $\mathbb{Z}^{2}$ (i.e., $\mathbb{B}^{2}$ ), Theorem 1(ii) holds with $q(p, 2)=c^{\prime} p^{2} \log ^{-2} p^{-1}$ for some absolute constant $c^{\prime}$, much better than the bound $\exp \left(-C^{\prime \prime} / p\right)$ that results from bootstrap percolation arguments. See Section 6 for details. Using Theorems 2 and 3, we can also provide upper and lower bounds for the number of steps required for the game to terminate on $\mathbb{Z}^{2}$; see Section 6.

Matchings and independent sets. Our proofs of Theorems 2 and 3 rely on the following simple characterization of winning positions for Trap on a finite graph. A proof appears in [7], and a closely related result appears in [2]; for the reader's convenience, we will also present a proof.

Proposition 4. Let $G=(V, E)$ be a finite, connected, simple graph. Trap on $G$ starting from $v \in V$ is a win for the first player if and only if $v$ is contained in all maximum-cardinality matchings of $G$.

Now we consider Vicious Trap. Recall that this game is the same as Trap except that in addition to moving the token along an edge to a previously unvisited vertex, a player may delete any subset of the vertices that he could have moved to (i.e., of the neighbors of the previous vertex other than the current vertex). Moves to deleted vertices are forbidden, and a player who cannot move loses. For this game, we provide an analogous characterization of winning positions, now involving maximum-cardinality independent sets.

Proposition 5. Let $G=(V, E)$ be a finite, connected, simple graph. Vicious Trap on $G$ starting from $v \in V$ is a loss for the first player if and only if $v$ is contained in every maximum-cardinality independent set of $G$. 
Trap and Vicious Trap are equivalent on a bipartite graph $G$. This is because it is never advantageous for a player to delete vertices, since those vertices are inaccessible to the other player anyway. By Propositions 4 and 5, this gives an interesting proof that on a finite simple bipartite graph; a vertex is contained in all maximum matchings if and only if it is absent from some maximum independent set. This fact can be deduced more directly from König's theorem which states that on a bipartite graph, the number of edges in a maximum matching equals the number of vertices in a minimum vertex cover [3], Theorem 5.3.

Since the graphs we consider ( $d$-dimensional lattices and subgraphs thereof) are bipartite, the conclusions of Theorems 1-3 hold for Vicious Trap as well.

Simulations and conjectures. Proposition 4 gives rise to a practical algorithm for determining the outcome of Trap on a finite bipartite graph. We can find a maximum size matching $M$ using the Hopcroft-Karp algorithm [14]. Then we can search for all matched vertices $v$ from which there is no alternating path leading to an unmatched vertex. A vertex $v$ has this property precisely if it is contained in every maximum matching, that is, if it is a winning initial vertex for the first player.

To gain insight about Trap in the most interesting setting of $\mathbb{Z}^{2}$ with $p=q$, we may proceed as follows. Consider the square $[1, n]^{2} \cap \mathbb{Z}^{2}$, with odd and even vertices closed with equal probability $p=q$, and declare the game a draw if the token ever leaves the square. We may determine the outcome of this game by applying the method described above to the square with two different boundary conditions, and comparing the results. In one case, we modify the graph just outside the boundary of the square so that all internal boundary vertices of the resulting graph are even; this means that Eve wins if the token leaves the square. In the other case, we similarly arrange that Odin wins if the token leaves the square. An initial vertex should then be considered a draw if its outcomes differ between these two boundary conditions. Thus, we can identify the outcome from every initial vertex.

Figures 3 and 4 show the results of the above experiment on squares of sizes 50 and 400, with four different values of $p$. The results are suggestive of the following picture. For each $p$, there are domains within which one or other player can force a win, presumably owing to a local preponderance of closed vertices of the appropriate parity. These domains tend to abut each other, so that there are no regions of draws between them. (An additional complication is the appearance of "checkerboard" regions, from which the first player wins, near some interfaces between opposing domains.) The typical size of a domain apparently diverges as $p$ becomes small, and, if the square is not large enough to contain a whole domain, then instead draws are prevalent.

However, the simulations seem consistent with the hypothesis that the typical domain size is finite for each $p>0$, and only approaches $\infty$ as $p \rightarrow 0$. This would suggest that there are no draws on $\mathbb{Z}^{2}$ for any $p>0$. (Other interpretations of the data are possible, and our confidence in this conclusion is not especially high.) 


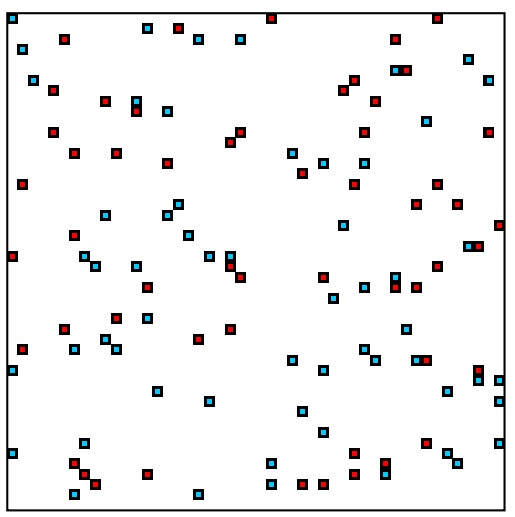

$p=0.05$

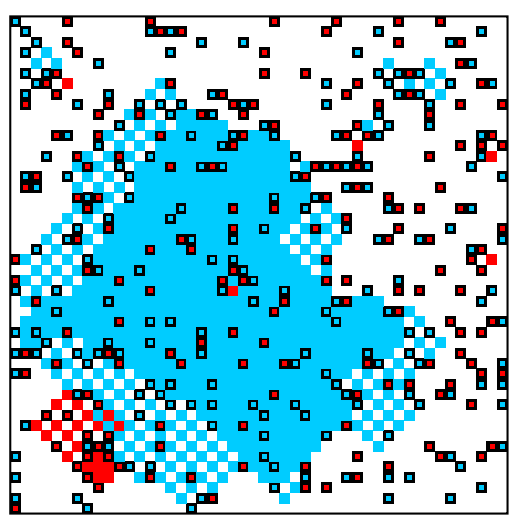

$p=0.15$

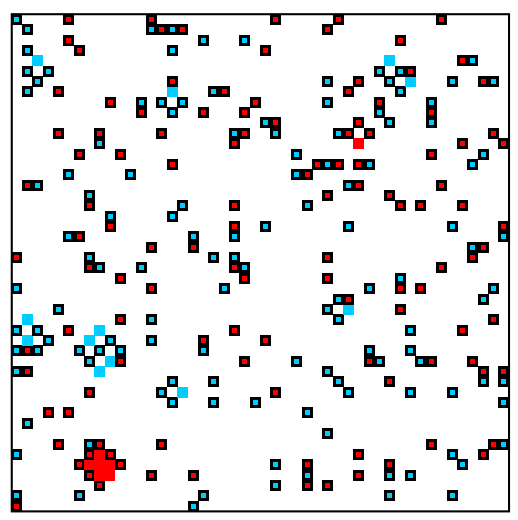

$p=0.1$

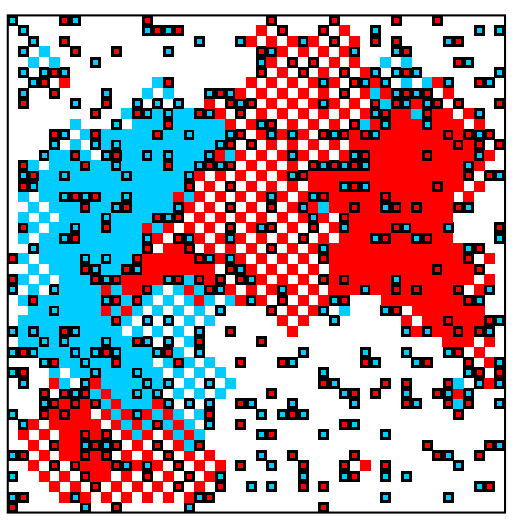

$p=0.2$

FIG. 3. Outcomes of Trap on a square of size $n=50$, with the game declared a draw if the token leaves the square. Closed vertices occur with probability $p$, and are outlined in black, with their interiors respectively blue or red according to whether they are odd or even (to reflect the fact that they are favorable to Eve or Odin respectively). Other vertices are blue if Eve wins, red if Odin wins, or white if the game is drawn, from that initial vertex.

On the other hand, on $\mathbb{Z}^{d}$ with $d \geq 3$, by analogy with the directed variants of Trap considered in [12], one may speculate that draws do occur when $p$ and $q$ are equal and sufficiently small.

Organization of the paper. The rest of this paper is organized as follows. In Section 2, we prove Theorem 1 using a bootstrap percolation argument. Next, in Section 3, we prove Propositions 4 and 5. In Section 4 and Section 5, respectively, we use these results to prove Theorems 3 and 2 on the diamond. In Section 6, we give some consequences for regions of other shapes, and for the length of the game on $\mathbb{Z}^{2}$. 


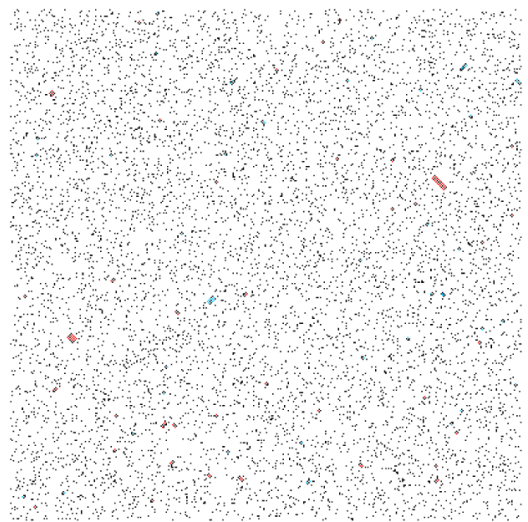

$p=0.05$

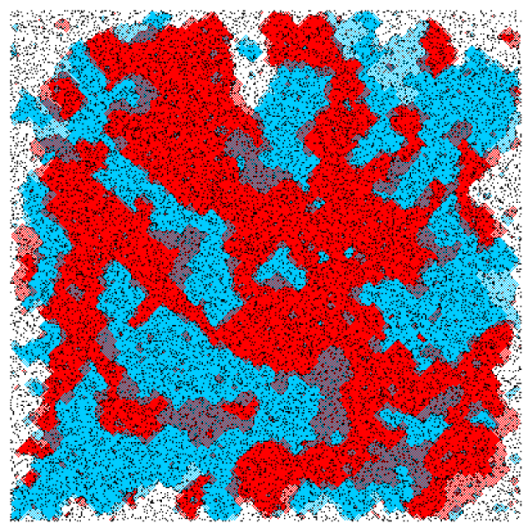

$p=0.15$

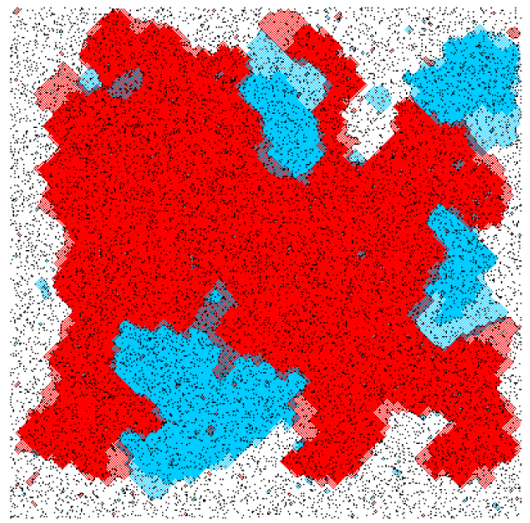

$p=0.1$

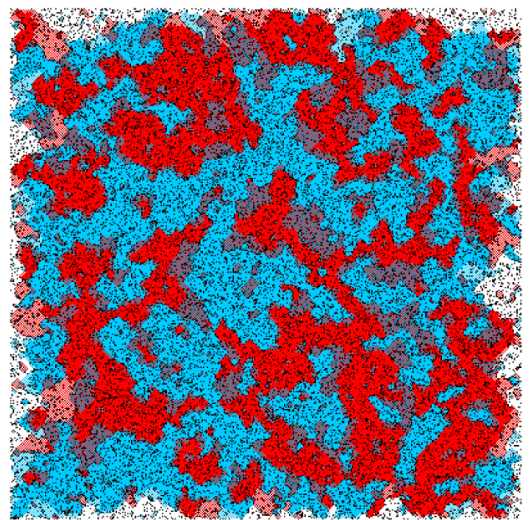

$p=0.2$

FIG. 4. Outcomes of Trap by initial vertex on a square of size $n=400$, with the boundary declared a draw. Closed vertices are black, wins for Eve are blue, wins for Odin are red and draws are white.

2. Bootstrap percolation bound. Our proof of Theorem 1 exploits a connection with a variant of bootstrap percolation. The basic idea is as follows. Let $u$ be an open even vertex of the body-centered lattice $\mathbb{B}^{d}$, and suppose that of the $2^{d}$ adjacent odd vertices, exactly one, $v$, is open. Starting from $v$, Eve can win immediately by moving to $u$. Thus, $v$ is effectively forbidden to Odin, and so we can now iterate the argument with $v$ added to the set of closed odd vertices.

Here is the relevant bootstrap percolation model on $\mathbb{Z}^{d}$, which we call the Fröbose model because it is a natural extension to $d$ dimensions of a model introduced in [8]. We start with a given subset $X_{0}$ of $\mathbb{Z}^{d}$, whose elements are said to be occupied at time 0 . We define the set of occupied vertices at time $t$, denoted $X_{t}$, for $t>0$, inductively as follows. Any vertex occupied at time $t-1$ remains occupied at time $t$. In addition, if all but one of the elements of any hypercube of the form $u+\{0,1\}^{d}$ are occupied at time $t-1$, then the one remaining vertex of the 
hypercube becomes occupied at time $t$. Let $\left\langle X_{0}\right\rangle=X_{\infty}:=\bigcup_{t=0}^{\infty} X_{t}$ be the set of eventually occupied vertices.

Given an initially occupied set $X_{0} \subseteq \mathbb{Z}^{d}$, a set $W \subseteq \mathbb{Z}^{d}$ is said to be internally spanned if $W \subseteq\left\langle X_{0} \cap W\right\rangle$, that is, if $W$ becomes fully occupied when we start from only the initially occupied vertices in $W$.

Let $B(n):=[1, n]^{d} \cap \mathbb{Z}^{d}$. The following is a standard result of bootstrap percolation, adapted to the Fröbose model.

PROPOSITION 6. Fix $p$ and let each vertex of $\mathbb{Z}^{d}$ be initially occupied independently with probability $p$. For all $d \geq 1$ and $p>0$, we have

$$
\mathbb{P}(B(n) \text { is internally spanned }) \rightarrow 1 \quad \text { as } n \rightarrow \infty .
$$

Proposition 6 follows from arguments of [18]. Another version of the argument, giving much tighter bounds, appears in [11]. The relevant results in $[11,18]$ state that the conclusion of Proposition 6 holds for another model called modified bootstrap percolation (in which a vertex becomes occupied if it has at least one occupied neighbor in each dimension). The Fröbose model is "weaker" in the sense that it is harder for a vertex to become occupied, so the conclusion itself does not carry over directly. However, the proofs in $[11,18]$ proceed by defining particular events $\mathcal{E}_{n}$ such that $\mathbb{P}\left(\mathcal{E}_{n}\right) \rightarrow 1$ as $n \rightarrow \infty$, and such that $B(n)$ is internally spanned (with respect to the modified model) on the event $\mathcal{E}_{n}$. It turns out (and it is straightforward to verify) that $B(n)$ is also internally spanned with respect to the Fröbose model on the same event $\mathcal{E}_{n}$, so Proposition 6 immediately follows.

To connect the Fröbose bootstrap model with Trap on $\mathbb{B}^{d}$, let $\mathbb{B}_{\mathrm{o}}^{d}$ (resp., $\mathbb{B}_{\mathrm{e}}^{d}$ ) be the graph comprising all odd (even) vertices of $\mathbb{B}^{d}$, with an edge between any pair of vertices that are at $\ell_{1}$ distance 2 . Obviously, $\mathbb{B}_{0}^{d}$ is isomorphic to the standard hypercubic lattice $\mathbb{Z}^{d}$. Let vertices of $\mathbb{B}^{d}$ be open or closed, as usual. Then we may run the Fröbose bootstrap percolation model on $\mathbb{B}_{\mathrm{o}}^{d}$ (by which we mean that we consider the image under the obvious isomorphism of the model on $\mathbb{Z}^{d}$ ). We declare the closed odd vertices initially occupied.

Recall that $B(n):=[1, n]^{d} \cap \mathbb{Z}^{d}$. For an odd vertex $u \in \mathbb{B}_{\mathrm{o}}^{d}$, define the set of odd vertices $\widetilde{B}_{\mathrm{O}}(u, n):=u+2 B(n) \subset \mathbb{B}_{\mathrm{o}}^{d}$. Note that the induced subgraph of $\widetilde{B}_{\mathrm{O}}(u, n)$ in the graph $\mathbb{B}_{\mathrm{o}}^{d}$ is isomorphic to the induced subgraph of $B(n)$ in $\mathbb{Z}^{d}$. Also let $\widetilde{B}_{\mathrm{e}}(u, n) \subset \mathbb{B}_{\mathrm{e}}^{d}$ be the set of all even vertices all of whose neighbors lie in $\widetilde{B}_{\mathrm{o}}(u, n)$, and let $\widetilde{B}(u, n)=\widetilde{B}_{\mathrm{o}}(u, n) \cup \widetilde{B}_{\mathrm{e}}(u, n)$.

We call the box $\widetilde{B}(u, n) \operatorname{good}$ if (i) all of its even vertices are open, and (ii) its odd subgraph $\widetilde{B}_{\mathrm{O}}(u, n)$ is internally spanned with respect to the Fröbose bootstrap model on $\mathbb{B}_{\mathrm{O}}^{d}$ started with the closed vertices occupied.

Proposition 7. Suppose that the box $\widetilde{B}(u, n)$ is good. Then from every odd $v \in \widetilde{B}(u, n)$, Eve has a winning strategy for Trap that guarantees that the token never leaves $\widetilde{B}(u, n)$. 
Proof. Let $X_{0}$ be the set of closed odd vertices in $\widetilde{B}_{\mathrm{o}}(u, n)$, and let $X_{t} \subseteq$ $\widetilde{B}_{\mathrm{O}}(u, n)$ be the set of vertices that are occupied at time $t$ starting from $X_{0}$ occupied. For $v \in \widetilde{B}_{\mathrm{o}}(u, n)$, let $T(v)$ be the time at which $v$ becomes occupied, that is, let $T(v)=t$ if $v \in X_{t} \backslash X_{t-1}$.

For the purpose of this proof, it is convenient to allow Odin to move to a closed vertex, but declare an immediate win for Eve if he does so. (This clearly does not change the outcome of the game.) We claim that starting from any $v \in \widetilde{B}_{\mathrm{o}}(u, n)$, Eve has a winning strategy that guarantees that $T$ is strictly decreasing along the sequence of odd vertices that are visited. Here is Eve's strategy, which we define inductively. Suppose (perhaps after some steps of the game played according to such a strategy) that it is Eve's turn. Then the token is at an odd vertex $v$; suppose $T(v)=t \geq 1$. By definition of the Fröbose model, there exists an even neighbor $w$ of $v$, all of whose neighbors other than $v$ lie in $X_{t-1}$. Since $v$ is the first vertex in $X_{t}$ that has been visited, no other neighbor of $w$ has been visited before, therefore, $w$ has not been visited. Therefore, Eve moves to $w$, and Odin must then move to an element of $X_{t-1}$, as required.

In particular, we deduce that starting from a vertex in $X_{t}$, Eve has a winning strategy that guarantees that the token remains in $\widetilde{B}(u, n)$ and that Eve makes at most $t$ moves.

Standard results also show that $\mathbb{Z}^{d}$ itself is internally spanned almost surely in the Fröbose model for any positive density $p$ of initially occupied sites. Hence, a minor variant of the above argument already shows that with $p>0$ and $q=0$, Eve wins on $\mathbb{B}^{d}$ if she has the first move. To deal with small positive $q$ and the possibility that Odin starts, we need to be a little more careful.

Proof of Theorem 1. Let $0:=(0, \ldots, 0) \in \mathbb{B}_{\mathrm{e}}^{d}$ and $\iota:=(1, \ldots, 1) \in \mathbb{B}_{\mathrm{o}}^{d}$. By translation invariance, it suffices to prove the claims for Trap started at 0 or $\iota$. For an integer $n \geq 1$ and $x \in \mathbb{Z}^{d}$, we introduce the renormalized box $\widehat{B}(x, n):=$ $\widetilde{B}(\iota+2 n x, n)$. Note that these boxes are disjoint for different $x$, but adjacent boxes almost abut each other: if $\|x-y\|_{1}=1$ then there is a layer of even vertices between $\widehat{B}(x, n)$ and $\widehat{B}(y, n)$, but all neighbors of those vertices lie in one of the two boxes.

We first prove part (ii). Let $p_{\mathrm{c}}$ be the critical probability of site percolation on the $d$-dimensional star-lattice, that is, the graph with vertex set $\mathbb{Z}^{d}$ and an edge between $u$ and $v$ whenever $\|u-v\|_{\infty}=1$. Fix $p>0$. By Proposition 7 , there exists $n \geq 1$ such that with $q=0$, we have $\mathbb{P}(\widehat{B}(0, n)$ is good $)>1-p_{\mathrm{c}}$. Since the box has finitely many even vertices, the same conclusion holds for $q$ sufficiently small; fix such a $q$.

Let $Z:=\left\{x \in \mathbb{Z}^{d}: \widehat{B}(x, n)\right.$ is not good $\} \cup\{0\}$. Note that we include the origin regardless of whether or not $\widehat{B}(0, n)$ is good. The set $Z$ induces a subgraph of the star-lattice; let $K$ be the vertex set of the component containing 0 . Then $K$ is finite almost surely. Let $S$ be the union of the renormalized boxes $\widehat{B}(x, n)$ for $x \in K$, 


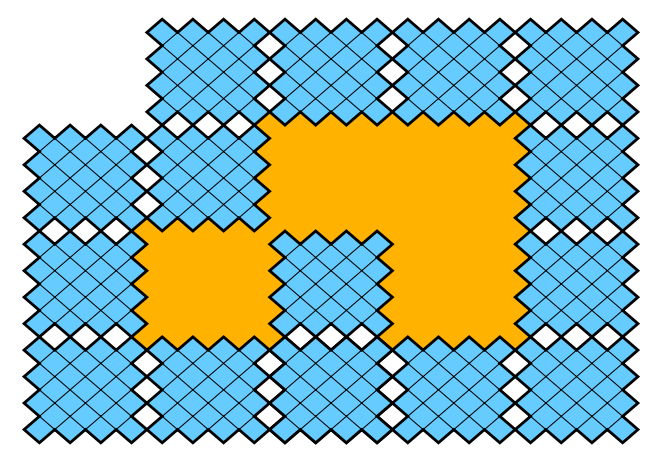

FIG. 5. A finite region $S$ surrounded by good boxes. (Vertices of $\mathbb{B}^{2}$ are shown as squares at 45 degrees to the axes.) Odin can only leave $S$ by entering a good box, whereupon Eve can win.

together with the set of all even vertices of $\mathbb{B}^{d}$ adjacent to them. Then $S$ is a.s. finite and contains $\widehat{B}(0, n)$ (which contains 0 and $\iota$ ). Furthermore, every infinite path in $\mathbb{B}^{d}$ starting from a vertex in $S$ intersects some good box that is not $\widehat{B}(0, n)$. See Figure 5.

Suppose that the token starts from 0 or $\iota$ and at some point leaves $S$. Then it must do so by entering a good box, and it must enter it at an odd vertex, via a move of Odin. Proposition 7 implies that Eve can then win within that box. It follows that the outcome of Trap starting from 0 or $\iota$ is identical to the outcome restricted to $S$ (i.e., with moves out of $S$ forbidden, and the same vertices closed as before). Since $S$ is finite, the game cannot be a draw, thus establishing (ii).

Now we prove (i). For all $p>0$, the conclusion of (ii) applies when $q=0$, so the game is not a draw. However, Eve has a simple strategy that guarantees she cannot lose: she always moves in direction $(1, \ldots, 1)$ (the relevant vertex cannot be closed, and cannot have been previously visited). Therefore, Odin cannot have a winning strategy, so Eve wins.

As mentioned in the Introduction, the method of this section could likely be extended to obtain the conclusions of Theorem 1 for Trap on the Euclidean lattice $\mathbb{Z}^{d}$ for all $d$. The main required step is to show the analogue of Proposition 6 for the following variant bootstrap model defined on the odd vertices of $\mathbb{Z}^{d}$ (i.e., vertices whose coordinates have odd sum): if all but one of the $2 d$ odd neighbors of any even vertex are occupied, then the final odd neighbor becomes occupied at the next step.

We emphasize that the bootstrap argument of this section is not tight. For example, in the bottom-right picture of Figure 2, Eve wins although the initial vertex does not become occupied in the bootstrap model. To get better bounds, we turn our attention to matchings.

3. Matchings and independent sets. In this section, we prove Propositions 4 and 5. Throughout this section $G=(V, E)$ will be a finite, connected, simple, 
undirected graph. For $W \subseteq V$, let $G \backslash W$ denote the subgraph of $G$ induced by $V \backslash W$.

A matching $M$ of $G$ is a set of edges of $G$ no two of which share a vertex. We say that $M$ matches a vertex $v$, or that $v$ is matched, if $v$ is incident to some edge of $M$. In that case, the other incident vertex to this edge is called the partner of $v$. An independent set is a set of vertices no two of which are adjacent. By a maximum matching or independent set, we mean one of maximum cardinality.

As mentioned in the Introduction, Proposition 4 is proved in [7]; for completeness, we also present a proof.

ProOf of Proposition 4. In a game of Trap on $G$, if the first move is from $v$ to $w$ then the remainder of the game is clearly equivalent to Trap on $G \backslash\{v\}$ with initial vertex $w$. We will prove the claimed result by induction on the number of vertices of $G$. In the base case $V=\{v\}$, the vertex $v$ is not in the maximum matching, and indeed the first player loses.

Suppose that $v$ lies in every maximum matching of $G$, and fix one such matching $M$. Let $w$ be the partner of $v$ in $M$, and let the first player move to $w$. We claim that $M^{\prime}:=M \backslash\{\{v, w\}\}$ is a maximum matching of $G \backslash\{v\}$ (so that in particular $w$ does not lie in every maximum matching of this graph). Indeed, $M^{\prime}$ is clearly a maximum matching of $G \backslash\{v, w\}$. Any larger matching $M^{\prime \prime}$ of $G \backslash\{v\}$ must therefore contain $w$. But from such an $M^{\prime \prime}$, we could obtain a maximum matching of $G$ with $v$ not matched by matching $w$ to $v$ instead of its partner in $M^{\prime \prime}$. This is a contradiction, proving the claim. Now by the inductive hypothesis, the next player loses on $G \backslash\{v\}$ starting from $w$. Thus, the first player wins on $G$ starting from $v$.

Now suppose that $v$ does not lie in every maximum matching of $G$. Suppose that the first player moves to any neighbor $w$ of $v$. We claim that $w$ lies in every maximum matching of $G \backslash\{v\}$. A maximum matching of $G$ that does not contain $v$ is also a maximum matching of $G \backslash\{v\}$, so maximum matchings of $G$ and $G \backslash\{v\}$ have the same size. But if $M$ is a maximum matching of $G \backslash\{v\}$ that does not contain $w$, then $M \cup\{\{v, w\}\}$ is a larger matching of $G$, a contradiction. This proves the claim. Now by the inductive hypothesis, the next player wins on $G \backslash\{v\}$ starting from $w$. But $w$ was an arbitrary neighbor of $v$, so the first player loses on $G$.

ProOF OF Proposition 5. The proof is again by induction on the number of vertices. In the base case $V=\{v\}$, the vertex $v$ lies in every maximum independent set, and indeed the first player loses Vicious Trap.

Suppose that there exists a maximum independent set that does not contain $v$. Any such set must contain a neighbor of $v$, otherwise it could be enlarged by adding $v$. From among the maximum independent sets that do not contain $v$, let $I$ be one that contains the fewest possible neighbors of $v$. Let the first player destroy the set $W$ of all neighbors of $v$ that do not lie in $I$, and move to some other neighbor $w \in I$. We claim that every maximum independent set of $G \backslash(W \cup\{v\})$ contains $w$. 
Indeed, $I$ is such a set, and any other independent set of the same size would also be a maximum independent set of $G$ containing fewer neighbors of $v$, contradicting the choice of $I$. By the inductive hypothesis, the next player loses. Therefore, the first player wins.

Now suppose that $v$ lies in every maximum independent set of $G$. Let $I$ be a maximum independent set of $G$. Then $I^{\prime}:=I \backslash\{v\}$ is a maximum independent set of $G \backslash\{v\}$. (Indeed, if $I^{\prime \prime}$ is a larger independent set of $G \backslash\{v\}$ then either it contains no neighbor of $v$, in which case it is an independent set of $G$ not containing $v$, or else adding $v$ gives an independent set of $G$ that is larger than $I$.) Now, if the first player destroys a set $W$ of neighbors of $v$ and moves to $w \notin W$, then $I^{\prime}$ is also a maximum independent set of $G \backslash(W \cup\{v\})$. Since $I^{\prime}$ does not contain $w$, the next player wins, by the inductive hypothesis.

4. Upper bound: Matching all odd vertices. In this section, we prove Theorem 3, which states that Eve typically wins Trap on the diamond $D_{n}$ if $n$ is sufficiently large as a function of $p$. This will be proved via Proposition 4 , by showing that there exist appropriate matchings in $D_{n}$.

Throughout, we suppose that each odd vertex of the diamond $D_{n}$ is closed with probability $p$, independently for different vertices, and all even vertices are open. We denote the associated probability measure $\mathbb{P}=\mathbb{P}_{p}$. It will be convenient to consider arbitrary (partial) matchings of the diamond $D_{n}$ itself, not just of its open subgraph. Edges incident to closed vertices in such a matching will be irrelevant in the eventual application to the game. Here is the key result of this section.

Proposition 8. There exists $C>0$ and an event $\mathcal{E}=\mathcal{E}_{n}$ such that if $p \rightarrow 0$ and $n \rightarrow \infty$ in such a way that $n>\left(C \log p^{-1}\right) / p$ then we have $\mathbb{P}_{p}\left(\mathcal{E}_{n}\right) \rightarrow 1$, and such that on $\mathcal{E}_{n}$ we have the following:

(i) There exists a matching $M$ of $D_{n}$ that matches all open odd vertices.

(ii) For every protected even vertex $v$, there exists a matching $M_{v}$ in $D_{n}$ that matches all open odd vertices but leaves $v$ unmatched.

Here is some motivation for Proposition 8. The diamond $D_{n}$ has $(2 n)^{2}$ odd vertices but only $(2 n-1)^{2}$ even vertices. Therefore, if we are to match all open odd vertices as in (i), then at least $4 n-1$ odd vertices must be closed. For this to happen with high probability, we certainly require that $p>1 / n$. Proposition 8 states that it suffices to take $p$ larger than this bound by a logarithmic factor, and that in that case we also get the stronger conclusion (ii). See Proposition 11 in the next section for a complementary result in the other direction.

For the proofs, it will be convenient to introduce an alternative coordinate system. We think of the diamond $D_{n}$ as rotated 45 degrees clockwise, so that $(2 n-1,0)$ is the bottom-right corner and $(-2 n+1,0)$ is the top-left corner. The following notation reflects this convention. For $i=-2 n+1,-2 n+2, \ldots, 2 n-1$, 


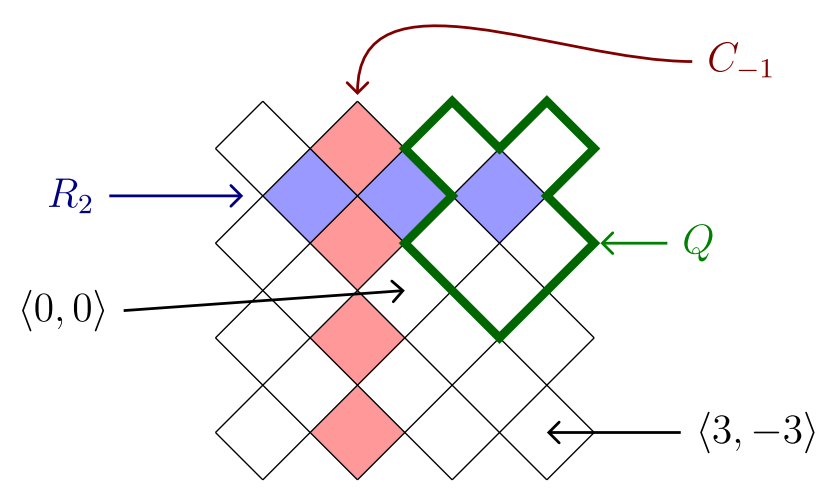

FIG. 6. The diamond $D_{2}$ : coordinates, an even row, an odd column and the top-right quadrant.

let $C_{i}=\left\{(x, y) \in D_{n}: x+y=i\right\}$ be the $i$ th column of the diamond. Notice that if $i$ is odd then $C_{i}$ comprises $2 n$ odd vertices, while if $i$ is even then $C_{i}$ comprises $2 n-1$ even vertices. We shall call the two cases odd columns and even columns, respectively. Similarly, for $j=-2 n+1, \ldots, 2 n-1$, let $R_{j}=\{(x, y) \in$ $\left.D_{n}: y-x=j\right\}$ be the $j$ th row (which may again be odd or even). If $i$ and $j$ have the same parity, then $C_{i}$ and $R_{j}$ intersect in a unique vertex, which we write as

$$
\langle i, j\rangle:=\left(\frac{i-j}{2}, \frac{i+j}{2}\right) .
$$

(If $i$ and $j$ have opposite parity, $C_{i}$ and $R_{j}$ do not intersect). See Figure 6 .

We divide the diamond into quadrants. The top-right quadrant is given by

$$
Q=Q^{0}=Q_{n}^{0}:=\left(\bigcup_{i=0}^{2 n-1} R_{i}\right) \cap\left(\bigcup_{i=1}^{2 n-1} C_{i}\right),
$$

that is, the vertices in top $2 n$ rows and the rightmost $2 n-1$ columns. For $k=1,2,3$ we define the quadrant $Q^{k}=\theta^{k}(Q)$, where $\theta$ is the anticlockwise rotation by 90 degrees about the origin. Notice that the diamond can be written as the disjoint union

$$
D_{n}=\{\langle 0,0\rangle\} \cup \bigcup_{k=0}^{3} Q^{k} .
$$

Given a matching $M$ in a graph, we say that a directed path $\left(v_{0}, v_{1}, \ldots, v_{\ell}\right)$ is $M$-alternating if it is self-avoiding, and every other edge of the path starting with the first one belongs to $M$, that is, $\left\{v_{i}, v_{i+1}\right\} \in M$ for all even $i$. We will use the following simple fact. Recall that we allow matchings to include closed vertices.

LEMMA 9. Let $G$ be a finite bipartite graph in which the odd vertices are declared open or closed. Let $M$ be a matching that matches all open odd vertices. 
Let $v$ be an even vertex that is matched in $M$. If there is an $M$-alternating path starting from $v$ that contains a closed odd vertex, then there exists a matching $M_{v}$ that matches all open odd vertices but does not match $v$.

ProOF. Let $\left(v_{0}, v_{1}, \ldots\right)$ be an $M$-alternating path starting at $v=v_{0}$, and let $v_{2 \ell+1}$ be the first closed odd vertex on the path. Construct $M_{v}$ from $M$ by removing the edges $\left\{v_{0}, v_{1}\right\},\left\{v_{2}, v_{3}\right\}, \ldots,\left\{v_{2 \ell}, v_{2 \ell+1}\right\}$ and adding the edges $\left\{v_{1}, v_{2}\right\},\left\{v_{3}, v_{4}\right\}, \ldots,\left\{v_{2 \ell-1}, v_{2 \ell}\right\}$.

The following is the main step of our proof of Proposition 8.

LEMMA 10. Let $\mathcal{F}=\mathcal{F}_{n}$ be the event that in each odd row $R_{j} \cap Q$ of the quadrant $Q$, there is a closed vertex $\langle i, j\rangle$ that is not at either extreme end, that is, $i \notin\{1,2 n-1\}$. On $\mathcal{F}$, there exists a matching $M$ of $Q$ with the following properties:

(i) All open odd vertices are matched.

(ii) For each protected even vertex $v \in Q$, either:

(a) there exists an $M$-alternating path in $Q$ from $v$ to the top-left corner $\langle 1,2 n-1\rangle$ of $Q$, or

(b) there exists an $M$-alternating path in $Q$, containing a closed vertex, from $v$ to the top-right corner $\langle 2 n-1,2 n-1\rangle$ of $Q$.

(iii) Each vertex $\langle 2 n-1, j\rangle$ in the rightmost column $Q \cap C_{2 n-1}$ is matched in the down-left direction, to $\langle 2 n-2, j-1\rangle$.

ProOF. The construction is illustrated in Figure 7. On $\mathcal{F}$, fix a set $H$ of closed vertices as follows. For each $j \in\{1,3, \ldots, 2 n-1\}$, let $m_{j} \in\{3,5, \ldots, 2 n-3\}$ be the largest number such that $\left\langle m_{j}, j\right\rangle$ is closed, so that this is the rightmost closed vertex in the $j$ th row of $Q$ barring the rightmost column. [This choice will be important in the proof of property (ii).] Let $H=\left\{\left\langle m_{j}, j\right\rangle: j \in\{1, \ldots, 2 n-1\}\right\}$. We construct the matching $M$ in such a way that each vertex in an even row is matched to some vertex in the odd row above it, avoiding the vertices in $H$. Specifically, for each $j \in\{1, \ldots, 2 n-1\}$, if $i>m_{j}$ then $(\langle i, j\rangle,\langle i-1, j-1\rangle) \in M$; if $i<m_{j}$ then $(\langle i, j\rangle,\langle i+1, j-1\rangle) \in M$. Every odd vertex in $Q \backslash H$ is matched, so every open odd vertex is matched (as well as every even vertex and perhaps some closed odd vertices), so (i) holds. Since $H$ has no vertices in the rightmost column, (iii) holds.

We now proceed to check (ii). Fix a protected even vertex $\langle i, j\rangle \in Q$. So there exists a closed odd vertex $\left\langle i^{*}, j^{*}\right\rangle \in Q$ with $i^{*}>i$ and $j^{*}>j$. We consider two cases, which will correspond to the two cases in the conclusion of part (ii).

Case (a). There exists $\left\langle i^{*}, j^{*}\right\rangle \in H$ with $i^{*}>i$ and $j^{*}>j$. Let $\left\langle i^{*}, j^{*}\right\rangle$ be the lowest element of $H$ that is above and right of $\langle i, j\rangle$, that is, the vertex satisfying the above condition for which $j^{*}$ is smallest. For $j \leq k<j^{*}$, the even vertex $\langle i, k\rangle$ 


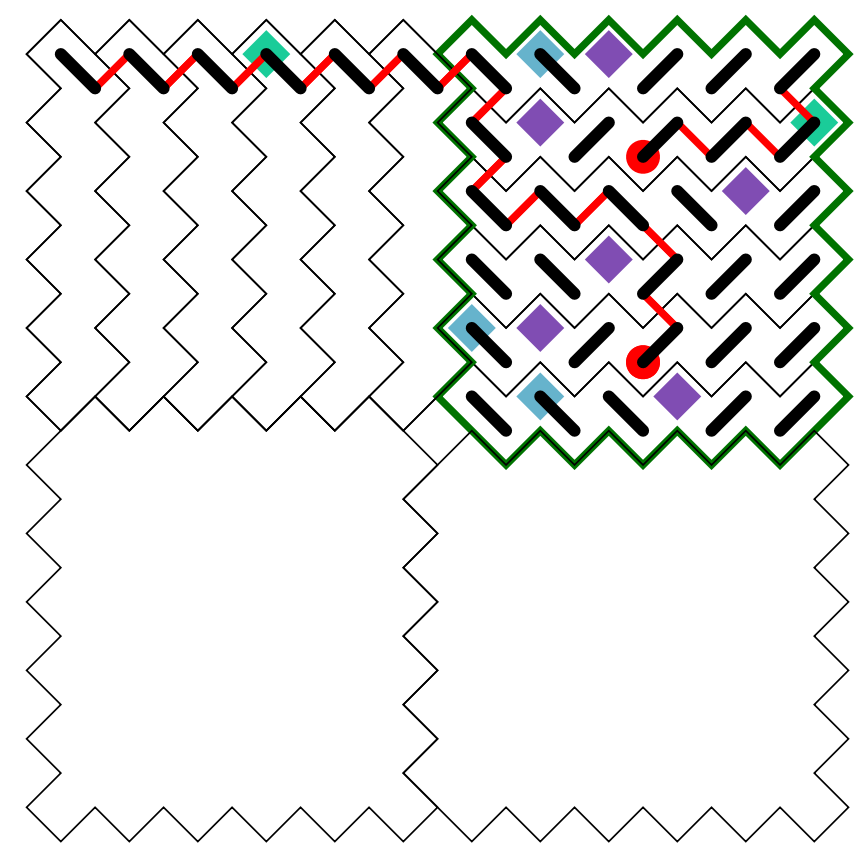

FIG. 7. Matching and alternating paths used in the proof of Lemma 10. The matching $M$ in the top-right quadrant $Q$ of the diamond $D_{6}$ is shown (together with the partition of the rows of $Q$ into pairs used in the construction of $M$ ). Closed vertices are shown as filled squares, with distinguished colors for those closed vertices comprising the set $H$, and those in the rightmost column. Alternating paths from two protected vertices (discs) are shown, ending at the top-right and top-left corners of the quadrant. In the latter case, an extension into the top-left quadrant is also shown. (Note that the alternating paths are used to construct further matchings, and are not directly related to trajectories of the token.)

is to the right of the element of $H$ in the row immediately above it, so it is matched in the up-right direction. Therefore, there is an $M$-alternating path $\pi_{1}$ from $\langle i, j\rangle$ to $\left\langle i, j^{*}-1\right\rangle$ consisting of alternate up-right and up-left steps. (The path is empty if $\left.j^{*}=j+1.\right)$

Since $\left\langle i, j^{*}-1\right\rangle$ is to the left of $\left\langle i^{*}, j^{*}\right\rangle$, it is matched in the up-left direction. Thus, there is an $M$-alternating path $\pi_{2}$ (again possibly empty) from $\left\langle i, j^{*}-1\right\rangle$ to $\left\langle 2, j^{*}-1\right\rangle$ consisting of alternate up-left and down-left steps.

Finally, since $H$ has no elements in the leftmost column of $Q$, there is an $M$ alternating path $\pi_{3}$ from $\left\langle 2, j^{*}-1\right\rangle$ to $\langle 1,2 n-1\rangle$ consisting of alternate up-left and up-right steps. Concatenating $\pi_{1}, \pi_{2}, \pi_{3}$ gives a path to the top-left corner of the quadrant, as required.

Case (b). There does not exist $\left\langle i^{*}, j^{*}\right\rangle \in H$ with $i^{*}>i$ and $j^{*}>j$. Recall that in each odd row, the element of $H$ is the rightmost closed odd vertex barring the rightmost column. Since $\langle i, j\rangle$ is protected, it must therefore be protected by a 
closed vertex in the rightmost column. Thus, there exists $j^{*}>j$ with $\left\langle 2 n-1, j^{*}\right\rangle$ closed.

Since $\langle i, j\rangle$ lies to the right of the element of $H$ in the row immediately above it, there is an $M$-alternating path from $\langle i, j\rangle$ to $\langle 2 n-1, j\rangle$ using alternate up-right and down-right steps, and thence to the top-right corner $\langle 2 n-1,2 n-1\rangle$ using alternate up-right and up-left steps. This path passes through the closed vertex $\left\langle 2 n-1, j^{*}\right\rangle$.

Proof of Proposition 8. Let $\mathcal{F}=\mathcal{F}_{n}$ be the event in Lemma 10. Also let $\mathcal{G}=\mathcal{G}_{n}$ be the event that the rightmost column $Q \cap C_{2 n-1}$ of the quadrant $Q$ contains a closed odd vertex. For $k=0, \ldots, 3$, let $\mathcal{F}^{k}=\mathcal{F}_{n}^{k}$ and $\mathcal{G}^{k}=\mathcal{G}_{n}^{k}$ be the images of $\mathcal{F}$ and $\mathcal{G}$ under the rotation $\theta^{k}$, that is, the corresponding events in the rotated quadrant $Q^{k}$. We take

$$
\mathcal{E}=\mathcal{E}_{n}:=\bigcap_{k=0}^{3}\left(\mathcal{F}^{k} \cap \mathcal{G}^{k}\right) .
$$

We first show that on $\mathcal{E}$, the properties (i) and (ii) in the statement of the proposition hold. The event $\mathcal{F}^{k}$ guarantees the existence of a matching $M^{k}$ of the quadrant $Q^{k}$, the image under the rotation $\theta^{k}$ of the matching in Lemma 10. Let $M:=\bigcup_{k=0}^{3} M^{k}$. Since the origin is even, $M$ matches all odd vertices, as required for (i). Let $v \in D_{n}$ be an even vertex. If $v$ is the origin then it is unmatched in $M$, as required for (ii). Otherwise, by symmetry we may assume $v \in Q$. By Lemma 10(ii) there exists an $M$-alternating path from $v$ that either contains a closed vertex, or ends at the top-left corner of $Q$. But in the latter case, we can extend this path along the top edge of $Q^{1}$ using alternate down-left and up-left steps, by Lemma 10(iii), as in Figure 7. Since $\mathcal{G}^{1}$ holds, the resulting path then also contains a closed vertex. Now applying Lemma 9 gives a matching that matches all open odd vertices but leaves $v$ unmatched, as required for (ii).

It remains to estimate $\mathbb{P}_{p}\left(\mathcal{E}_{n}\right)$. By a union bound, we get that $\mathbb{P}_{p}\left(\overline{\mathcal{F}_{n}}\right) \leq n(1-$ $p)^{n-2}$. Also, $\mathbb{P}_{p}\left(\overline{\mathcal{G}_{n}}\right)=(1-p)^{n}$. Therefore,

$$
\mathbb{P}_{p}\left(\mathcal{F}_{n} \cap \mathcal{G}_{n}\right) \geq 1-(n+1)(1-p)^{n-2} \geq 1-2 n e^{-p n / 2},
$$

provided $n \geq 4$. For each fixed $p$, the expression on the right-hand side of the last inequality is increasing in $n$ for $n>2 / p$. Hence for fixed $C$, if $p$ is sufficiently small and $n>\left(C \log p^{-1}\right) / p$, we have

$$
\mathbb{P}_{p}\left(\mathcal{F}_{n} \cap \mathcal{G}_{n}\right) \geq 1-\frac{2 C \log p^{-1}}{p} \exp \frac{-C \log p^{-1}}{2},
$$

which tends to 1 as $p \rightarrow 0$ provided $C>2$. By rotational symmetry, the probabilities $\mathbb{P}_{p}\left(\mathcal{F}^{k} \cap \mathcal{G}^{k}\right)$ are equal for $k=0, \ldots, 3$, so another union bound shows that $\mathbb{P}_{p}\left(\mathcal{E}_{n}\right) \rightarrow 1$. 
The proof of Theorem 3 is now straightforward.

Proof of Theorem 3. Choose $C$ as in Proposition 8, and let $p \rightarrow 0$ and $n>\left(C \log p^{-1}\right) / p$. On $\mathcal{E}_{n}$, let $M$ be the matching of Proposition 8(i). Modify $M$ by removing from the matching each edge that is incident to a closed vertex, to give a matching of the open subgraph of $D_{n}$. Since every open odd vertex is matched, this is a maximum matching, and every maximum matching matches all open odd vertices. By Proposition 4, we deduce that Eve wins from every open odd site. Recall also that by convention Eve also wins starting from a closed odd site.

Similarly, on $\mathcal{E}_{n}$, consider a protected even vertex $v$, and the associated matching $M_{v}$ from Proposition 8(ii). Removing edges incident to closed vertices as before gives a maximum matching in which $v$ is unmatched, so Proposition 4 gives that Eve wins from $v$.

5. Lower bound: Matching all even vertices. In this section, we prove Theorem 2. We adopt the same coordinate system for the diamond $D_{n}$ as in the last section, and we again consider the measure $\mathbb{P}_{p}$ under which odd vertices of $D_{n}$ are closed with probability $p$, and all even vertices are open. The following is the main result in this section.

Proposition 11. Take any $c>0$ and let $n<c /\left(p \log p^{-1}\right)$ with $p \rightarrow 0$. There exists an event $\mathcal{O}=\mathcal{O}_{n}$ with $\mathbb{P}_{p}\left(\mathcal{O}_{n}\right) \rightarrow 1$, such that on $\mathcal{O}_{n}$, for every odd vertex $v \in D_{n}$ there exists a matching $M_{v}$ of $D_{n}$ in which all even vertices are matched, but neither $v$ nor any closed odd vertex is matched.

The proof of Proposition 11 will use the next lemma. In the application, the set $H$ will consist of the closed vertices together with one additional arbitrary odd vertex $v$. The lemma will in fact be applied twice: both to rows and to columns. By an interval of $s$ consecutive rows, we mean a set of the form $\bigcup_{j=1}^{s} R_{j+a}$, and by an interval of $s$ consecutive odd rows we mean a set of the form $\bigcup_{j=1}^{S} R_{2 j+2 a+1}$, where $a \in \mathbb{Z}$.

LEMMA 12. Let $n$ and $s$ be positive integers. Let $H$ be a set of odd vertices of the diamond $D_{n}$. Suppose that every interval of $s$ consecutive odd rows contains at most $s$ vertices of $H$, and that for each $\langle i, j\rangle \in H$, there is no other $\left\langle i, j^{\prime}\right\rangle \in H$ in the same column with $\left|j-j^{\prime}\right|<2 s$. Then there exists a matching of $D_{n}$ that matches all even vertices but leaves $H$ unmatched.

Note that in the above lemma we do not require that $s \leq 2 n$. If $s>2 n$, then the first condition in the lemma is vacuously satisfied, and the second condition states that no odd column contains two or more elements of $H$. In the main case of interest, we will in fact choose $s$ to be approximately $\log p^{-1} / \log \log p^{-1}$. Lemma 12 will in turn be proved by partitioning the rows into suitable intervals, and using the following technical lemma. 
LEMMA 13. Fix $n$, and consider an interval of rows of the diamond

$$
W:=\bigcup_{j=a}^{b} R_{j} \subseteq D_{n},
$$

where $a \leq b$ and $a$ is odd. Let $H$ be a subset of the odd vertices of $W$, such that each column contains at most one vertex of $H$. Suppose that either:

(i) $b$ is odd, or

(ii) $b$ is even, and for each integer $t$, the top $t$ odd rows of $W$ contain at most $t$ vertices of $H$.

Then there exists a matching of $W$ that matches all even vertices but leaves $H$ unmatched.

ProOf. See Figure 8. The proof is by induction on $s:=\lfloor(b-a) / 2\rfloor$, the number of even rows of $W$. The inductive step will involve removing the bottom two rows of $W$ and modifying $H$. When $s=0$, either $W$ consists of one odd row [case (i)], or $W$ is empty [case (ii)]; in either case we take the empty matching.

Now suppose $s \geq 1$. Let $z=\langle\ell, a\rangle$ be the leftmost vertex of $H$ in the bottom row $R_{a}$. (If none exists, take $\ell=\infty$.) Consider any even site $u=\langle i, a+1\rangle$ in the next row $R_{a+1}$. If $u$ is to the left of $z$ (i.e., $i<\ell$ ), match it down-left to $\langle i-1, a\rangle$. If $u$ is to the right of $z$ (i.e., $i>\ell$ ), match it down-right to $\langle i+1, a\rangle$, unless the latter vertex is in $H$. In that case, match $u$ up-right to $\langle i+1, a+2\rangle$ instead. Since $H$ has at most one vertex in each column, this last vertex is not in $H$.

Let $W^{\prime}:=\bigcup_{j=a+2}^{b} R_{j}$, and let

$$
H^{\prime}:=\left(H \cap W^{\prime}\right) \cup\{\langle i+1, a+2\rangle: i>\ell \text { and }\langle i+1, a\rangle \in H\} .
$$

(Thus, $H \cap W^{\prime}$ is augmented by the set of odd vertices of $R_{a+2}$ that were already matched to vertices in $R_{a+1}$; this corresponds to shifting the vertices of $H$ in the

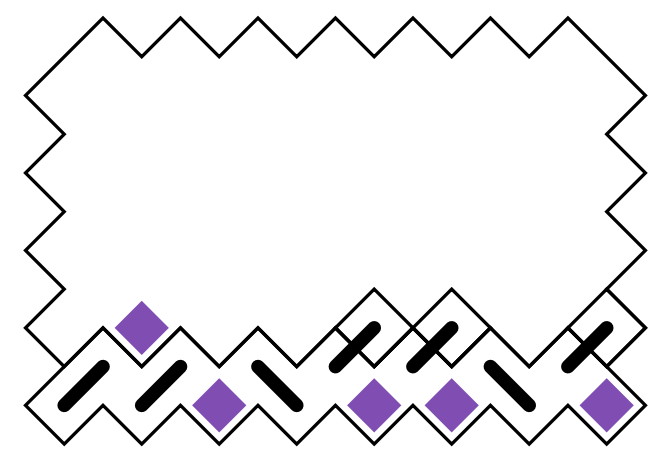

FIG. 8. Inductive step in the proof of Lemma 13 [in case (ii)]. Vertices of $H$ are shown as filled squares. The inductive hypothesis is applied after removing the bottom two rows, and modifying $H$ by adding the three outlined vertices. 
bottom row up by 2 , except for the leftmost one). We will apply the inductive hypothesis to $W^{\prime}$ and $H^{\prime}$, and combine the resulting matching with the matching constructed above.

To complete the argument, we must check that $W^{\prime}$ and $H^{\prime}$ indeed satisfy the conditions of the lemma. Since the construction of $H^{\prime}$ involves shifting vertices of $H$ vertically, $H^{\prime}$ still has no two vertices in the same column. If (i) holds for $W$, then (i) obviously holds for $W^{\prime}$ as well. If (ii) holds for $H$, we need only check condition (ii) for $H^{\prime}$ in the case $t=s-1$, that is, that $\left|H^{\prime}\right| \leq s-1$. If $\ell=\infty$, then $H^{\prime}=H \cap W^{\prime}$ and so this follows immediately from the condition on $H$. If $\ell<\infty$, then we have $|H| \leq s$, but the shifted vertex $\langle\ell, a+2\rangle$ corresponding to $z$ is not included in $H^{\prime}$, so $\left|H^{\prime}\right|=|H|-1 \leq s-1$.

ProOF OF LEMMA 12. We partition the rows of the diamond, starting from the bottom, into minimal intervals containing no more vertices of $H$ than odd rows. More precisely, let $\ell_{0}=-2 n+1$, and iteratively define $\ell_{k+1}$ to be the smallest odd integer in $\left(\ell_{k}, 2 n-1\right]$ for which

$$
\left|H \cap \bigcup_{j=\ell_{k}}^{\ell_{k+1}-1} R_{j}\right| \leq \frac{\ell_{k+1}-\ell_{k}}{2} ;
$$

if there is no such number then we instead take $\ell_{k+1}=2 n$, write $K:=k$, and stop the iteration.

Since any interval of $s$ rows contains at most $s$ vertices of $H$, each of these intervals must contain at most $s$ odd rows (i.e., $\ell_{k+1}-\ell_{k} \leq 2 s$ ) and, therefore, each of the corresponding regions $W_{k}:=\bigcup_{j=\ell_{k}}^{\ell_{k+1}-1} R_{j}$ contains no two vertices of $H$ in the same column. Furthermore, in each of these regions except perhaps the last, $W_{K}$, for every $t$, the top $t$ odd rows contain at most $t$ vertices of $H$ (otherwise, $\ell_{k+1}$ should have been smaller). On the other hand, the last region $W_{K}$ has an odd row at the top. Therefore, we can apply Lemma 13 to each of the regions $W_{0}, \ldots, W_{K}$ and take the union of the resulting matchings. (If $s \geq 2 n$, it is possible that $K=0$, and thus $W_{K}=D_{n}$.)

Proof of Proposition 11. Fix any $c>0$. Let

$$
s=\left\lceil\frac{4 \log p^{-1}}{\log \left[\left(\log p^{-1}\right) / 4 c\right]}\right\rceil .
$$

We define the following events. Let $\mathcal{R}=\mathcal{R}_{n, s}$ be the event that in any $s$ consecutive odd rows of $D_{n}$, at most $s-1$ vertices are closed. Let $\mathcal{T}=\mathcal{T}_{n, s}$ be the event that there are no two distinct closed vertices $\langle i, j\rangle$ and $\left\langle i, j^{\prime}\right\rangle$ in the same column with $\left|j-j^{\prime}\right|<2 s$. Finally, for an odd vertex $v=\langle i, j\rangle$ we define $\mathcal{X}_{v}=\mathcal{X}_{v, n, s}$ to be the event that there is no closed vertex $\left\langle i, j^{\prime}\right\rangle$ with $\left|j-j^{\prime}\right|<2 s$ and $j \neq j^{\prime}$.

Note that on $\mathcal{R} \cap \mathcal{T} \cap \mathcal{X}_{v}$, the hypothesis of Lemma 12 is satisfied when $H$ is taken to be the set of closed vertices together with $v$. (The addition of $v$ is the 
reason for the using $s-1$ in definition of $\mathcal{R}$.) Thus, there exists a matching $M_{v}$ satisfying the required conclusion for $v$. However, we cannot directly obtain the same conclusion simultaneously for all $v$ : indeed, the event $\mathcal{X}_{v}$ does not hold for vertices $v$ within distance $2 s$ of a closed vertex in the same column.

To address this issue, we also consider rotated versions of the same events. Let $\mathcal{R}^{\prime}, \mathcal{T}^{\prime}$ be the images of $\mathcal{R}, \mathcal{T}$ under the 90 degree anti-clockwise rotation $\theta$ about the origin. Also for an odd vertex $v=\langle i, j\rangle$, let $\mathcal{X}_{v}^{\prime}$ be the event that there is no closed vertex $\left\langle i^{\prime}, j\right\rangle$ with $\left|i-i^{\prime}\right|<2 s$ and $i \neq i^{\prime}$. By rotational symmetry, $v$ satisfies the required conclusion on $\mathcal{R}^{\prime} \cap \mathcal{T}^{\prime} \cap \mathcal{X}_{v}^{\prime}$ as well. It follows that on

$$
\mathcal{O}=\mathcal{O}_{n}:=\mathcal{R} \cap \mathcal{R}^{\prime} \cap \mathcal{T} \cap \mathcal{T}^{\prime} \cap \bigcap_{v \text { odd }}\left(\mathcal{X}_{v} \cup \mathcal{X}_{v}^{\prime}\right),
$$

the required conclusion holds for all odd vertices $v \in D_{n}$.

Now we estimate $\mathbb{P}_{p}\left(\mathcal{O}_{n}\right)$. For the following calculations, we always take $p$ to be sufficiently small such that $p^{-1}>c^{-1} \log p^{-1} / 4>5$ and $s<c /\left(p \log p^{-1}\right)$. Notice that this ensures that for all $n$ with $1 \leq n<c /\left(p \log p^{-1}\right)$ we have $n p<$ $1 / 20$, and that $s$ as given by (2) is at least 2 .

If $s>2 n$, then $\mathbb{P}_{p}\left(\mathcal{R}_{n, s}\right)=1$. Otherwise, a union bound over all intervals of $s$ consecutive odd rows gives

$$
\mathbb{P}_{p}\left(\mathcal{R}_{n, s}\right) \geq 1-2 n \mathbb{P}(Z \geq s-1) \geq 1-2 n \mathbb{P}(Z \geq s / 2),
$$

where $Z$ is a binomial random variable with parameters $(2 n s, p)$. Using $n p<$ $1 / 20$, a Chernoff bound then yields

$$
\mathbb{P}_{p}\left(\mathcal{R}_{n, s}\right) \geq 1-2 n(4 n p)^{s / 4} .
$$

The expression on the right is decreasing in $n$ for fixed $p$ and $s>0$. Hence, for $n<c /\left(p \log p^{-1}\right)$, using (2) we have

$$
\mathbb{P}_{p}\left(\mathcal{R}_{n, s}\right) \geq 1-\frac{2 c}{p \log p^{-1}}\left(\frac{4 c}{\log p^{-1}}\right)^{s / 4} \geq 1-\frac{2 c}{\log p^{-1}} .
$$

Now note that in any given odd column, the probability that there are two closed odd vertices with their vertical coordinates differing by less than $2 s$ is at most $2 n s p^{2}$. Taking a union bound over all odd columns, we obtain for $n<$ $c /\left(p \log p^{-1}\right)$,

$$
\mathbb{P}_{p}\left(\mathcal{T}_{n, s}\right) \geq 1-4 n^{2} s p^{2} \geq 1-\frac{4 c^{2} s}{\log ^{2} p^{-1}}
$$

Also, for each odd vertex $v$ we have $\mathbb{P}_{p}\left(\mathcal{X}_{v} \cup \mathcal{X}_{v}^{\prime}\right) \geq 1-(2 s p)^{2}$, and a union bound then gives that for $n<c /\left(p \log p^{-1}\right)$,

$$
\mathbb{P}_{p}\left(\bigcap_{v \text { odd }}\left(\mathcal{X}_{v} \cup \mathcal{X}_{v}^{\prime}\right)\right) \geq 1-4 n^{2}(2 s p)^{2} \geq 1-\frac{16 c^{2} s^{2}}{\log ^{2} p^{-1}} .
$$


Using the definition of $s$ in (2), we see that the right-hand sides of (4), (5), (6) each converge to 1 as $p \rightarrow 0$. By rotational symmetry, we have $\mathbb{P}_{p}\left(\mathcal{R}^{\prime}\right)=\mathbb{P}_{p}(\mathcal{R})$ and $\mathbb{P}_{p}\left(\mathcal{T}^{\prime}\right)=\mathbb{P}_{p}(\mathcal{T})$. A final union bound now shows that $\mathbb{P}_{p}\left(\mathcal{O}_{n}\right) \rightarrow 1$ as $p \rightarrow 0$ with $n<c /\left(p \log p^{-1}\right)$.

Proof of Theorem 2. We apply Proposition 11 . On the event $\mathcal{O}_{n}$, for each odd $v$, the matching $M_{v}$ is a maximum matching of the open subgraph of $D_{n}$, in which all even vertices are matched but $v$ is unmatched. By Proposition 4, Odin wins from every initial vertex, whether even or odd.

6. Further results. In this section, we justify the claims about protected vertices in the Introduction, and briefly address some consequences of Theorems 2 and 3 for Trap on finite regions other than the diamond, for the density of closed even vertices, and for the length of the game on $\mathbb{Z}^{2}$.

Protected vertices. We check the remarks following Theorem 3 regarding the set $S$. Write $L=C^{\prime} \log p^{-1} / p$, as in the definition of $S$ in (1). We adopt the rotated coordinate system of Section 4 . If $\langle i, j\rangle \in D_{n}$ satisfies $i, j \geq 0$ and $(2 n-i)(2 n-$ $j)>L$ then the probability that there is a closed vertex of $D_{n}$ above and right of $\langle i, j\rangle$ is at least

$$
1-(1-p)^{L} \geq 1-e^{-p L}=1-p^{C^{\prime}} .
$$

To ensure that all vertices in $S$ are protected, we need only check this condition (and similar ones involving other quadrants) for $O(L)$ vertices near the boundary of $S$. (A key point to note is that provided $2 n>L+2$, one of the vertices to be checked will be in the top row, of the form $\langle 2 n-L-\varepsilon, 2 n-1\rangle$ for some $\varepsilon \in(0,2]$.) Therefore, for $C^{\prime}>1$, a union bound shows that all vertices in $S$ are protected with high probability as $p \rightarrow 0$, uniformly in $n$.

On the other hand, we have as $p \rightarrow 0$, uniformly in $n$,

$$
\left|D_{n} \backslash S\right|=O\left(\int_{1}^{L} \frac{L}{x} d x\right)=O(L \log L)=O\left(p^{-1} \log ^{2} p^{-1}\right),
$$

as claimed.

Finite regions. Fix a finite connected region $A \subseteq \mathbb{Z}^{2}$ whose internal boundary consists entirely of odd vertices. Consider a game of Trap on the open subgraph of $A$, where as usual each odd vertex is closed with probability $p$. (Equivalently, we can consider Trap on $\mathbb{Z}^{2}$ started from a vertex in $A$, but declaring a win for Odin if Eve ever leaves $A$.) We can infer outcomes of this game in certain situations by comparing with the games played on smaller and larger diamonds and using Theorems 2 and 3. Suppose that $D_{n}^{\prime} \subseteq A \subseteq D_{N}^{\prime}$, where $D_{n}^{\prime}$ and $D_{N}^{\prime}$ are translates of diamonds $D_{n}$ and $D_{N}$. Starting from any vertex in $A$, if Odin wins the game 
played on $D_{N}$ then he wins the game on $A$ as well. If Eve wins in $D_{n}$ starting from a vertex in $D_{n}$, then she wins on $A$. However, the latter argument does not apply to initial vertices in $A \backslash D_{n}$.

As a concrete example, consider a square modified so that all internal boundary vertices are odd:

$$
B^{\#}(n):=\left([1, n]^{2} \cap \mathbb{Z}^{2}\right) \cup\left([0, n+1]^{2} \cap \mathbb{Z}_{\mathrm{o}}^{2}\right),
$$

where $\mathbb{Z}_{\mathrm{O}}^{2}$ is the set of odd vertices of $\mathbb{Z}^{2}$. In the following, $f(p) \gg g(p)$ means $f(p) / g(p) \rightarrow \infty$.

COROLlARY 14. Consider Trap on the open subset of $B^{\#}(n)$, where each odd vertex is closed with probability $p$ and all even vertices are open. If $p \rightarrow 0$ with $n<c /\left(p \log p^{-1}\right)$ and any $c$, then Odin wins from every vertex with high probability. If $p \rightarrow 0$ with $n \gg \log p^{-1} / p$, then with high probability, Eve wins from a proportion $1-o(1)$ of initial vertices.

PROOF. Since $B^{\#}(n)$ is contained in a translate of $D_{n+1}$, the first claim follows from Theorem 2. For the second claim, let $m=m(p)=2 C \log p^{-1} / p$ where $C$ is the constant of Theorem 3 . We can "approximately tile" $B^{\#}(n)$ with disjoint translates of $D_{m}$ in such a way that all but a proportion $o(1)$ of vertices are covered. By the law of large numbers, with high probability, the conclusion of Theorem 3 holds for a proportion $1-o(1)$ of these translates. Using the remarks following Theorem 3 about protected vertices, the required conclusion follows.

Density of closed even vertices. Recall that in dimension 2 , the body-centered lattice $\mathbb{B}^{2}$ and the square lattice $\mathbb{Z}^{2}$ are isomorphic. As mentioned in the Introduction, it is possible to give an alternative proof of Theorem 1 for $d=2$ using Proposition 8 , and this gives a much tighter bound on $q(p, 2)$ in part (ii) compared with the argument in Section 2.

COROLLARY 15. Let odd and even vertices of $\mathbb{Z}^{2}$ be closed with respective probabilities $p$ and $q$. There exists $c_{0}>0$ such that if $p>0$ and $q<$ $c_{0} p^{2} \log ^{-2} p^{-1}$, then almost surely, from every initial vertex, Trap is not a draw.

PROOF. For an even vertex $u \in \mathbb{Z}^{2}$, call a diamond-shaped region $D_{n}(u):=$ $u+D_{n} \operatorname{good}$ if it contains no closed even vertices, and it has a matching that matches all open odd vertices. Starting from any odd vertex in a good diamond $D_{n}(u)$, Eve can win within $D_{n}(u)$. We now proceed as in the proof of Theorem 1. [A diamond $D_{n}(u)$ induces a graph in $\mathbb{Z}^{2}$ isomorphic to that induced by a box $\widetilde{B}\left(u^{\prime}, n^{\prime}\right)$ in $\mathbb{B}^{2}$ as in Section 2.] It follows from Proposition 8 that if $q<c_{0} p^{2} \log ^{-2} p^{-1}$ for a suitable constant $c_{0}>0$, then choosing $n$ appropriately, the probability that $D_{n}(u)$ is good exceeds one minus the critical probability of the two-dimensional star-lattice. Almost surely, we can then surround the initial vertex by good diamonds, and the proof goes through as before. 
Length of the game. We can use Theorems 3 and 2 to obtain bounds on the time for Trap to terminate. For simplicity, we do this only for $q=0$, although analogous results are also available in the regime of the last result.

If Eve can win, then it is natural for her to try to win as quickly as possible, while Odin tries to prolong the game. Fix an initial vertex $v$, and let $\mathbf{E}$ and $\mathbf{O}$ denote the sets of all possible strategies for Eve and Odin respectively starting from $v$. For $\mathbf{e} \in \mathbf{E}$ and $\mathbf{o} \in \mathbf{O}$, let $T(\mathbf{e}, \mathbf{o})$ be the number of turns until the game terminates when Eve plays with strategy e and Odin plays with strategy o, provided Eve wins; if Eve does not win with this pair of strategies, let $T(\mathbf{e}, \mathbf{o})=\infty$. Define

$$
T=T_{v}:=\inf _{\mathbf{e} \in \mathbf{E}} \sup _{\mathbf{o} \in \mathbf{O}} T(\mathbf{e}, \mathbf{o}),
$$

that is, the minimum time in which Eve can guarantee to win.

COROLLARY 16. Let odd and even vertices of $\mathbb{Z}^{2}$ be closed with respective probabilities $p>0$ and $q=0$. There exist constants $c_{1}, C_{1}>0$ such that, for any initial vertex $v$, with high probability as $p \rightarrow 0$, the time $T=T_{v}$ for Eve to win Trap satisfies

$$
\frac{c_{1}}{p \log p^{-1}} \leq T \leq \frac{C_{1} \log ^{2} p^{-1}}{p^{2}}
$$

PROOF. By translation-invariance, we can assume without loss of generality that $v$ is $(0,0)$ or $(1,0)$. Let $n=\left\lfloor c /\left(p \log p^{-1}\right)\right\rfloor$ and $N=\left\lceil\left(C \log p^{-1}\right) / p\right\rceil$, where $c, C$ are the constants of Theorems 3 and 2. With high probability, Eve can guarantee a win without having to leave $D_{N}$, and hence she can win in at most $\left|D_{N}\right|$ moves. On the other hand, with high probability, Eve cannot win within $D_{n}$, and it takes at least $2 n-2$ steps for the token to leave $D_{n}$ starting from $v$.

\section{Open problems.}

(i) On $\mathbb{Z}^{d}$ with each vertex closed independently with probability $p$, does there exist $p>0$ for which Trap starting from the origin is a draw with positive probability? (It is plausible that the answer is no for $d=2$ and yes for $d \geq 3$.)

(ii) In the situation of (i) above, is the probability of a draw monotone in $p$ ?

(iii) On a diamond $D_{n}=\left\{u \in \mathbb{Z}^{2}:\|u\|_{1}<2 n\right\}$, with odd vertices closed with probability $p$ and all even vertices open, what happens within the window $c /\left(\log p^{-1}\right)<n p<C \log p^{-1}$ ? Is there a regime in which both players have substantial regions of winning initial vertices?

(iv) On $\mathbb{Z}^{2}$ with odd vertices closed with probability $p$ and all even vertices open, what more can be said about the minimum time $T$ in which Eve can guarantee a win? (Corollary 16 states that with high probability it is between $p^{-2}$ and $p^{-1}$ up to logarithmic factors.) Does $T$ converge when suitably scaled as $p \rightarrow 0$ ? 
(v) How do our results change on nonbipartite graphs such as the triangular lattice? How do the outcomes of Trap and Vicious Trap differ from each other?

(vi) How do our results change for the misère variant of Trap in which a player who cannot move wins, or for the variant game in which one specified player wins if either player cannot move? (See [13] for analysis of these games on random trees, and [16] for results on computational complexity of the misère game.)

Acknowledgments. Much of this work was completed at the Theory Group of Microsoft Research in Redmond during visits by James B. Martin and Johan Wästlund and an internship by Riddhipratim Basu. We thank Microsoft for its hospitality and support. We thank the referees for helpful suggestions.

\section{REFERENCES}

[1] Aizenman, M. and Grimmett, G. (1991). Strict monotonicity for critical points in percolation and ferromagnetic models. J. Stat. Phys. 63 817-835. MR1116036

[2] Anderson, W. N. JR. (1974). Maximum matching and the game of Slither. J. Combin. Theory Ser. B 17 234-239. MR0364014

[3] Bondy, J. A. and Murty, U. S. R. (1976). Graph Theory with Applications. American Elsevier Publishing Co., New York. MR0411988

[4] Cerf, R. and Cirillo, E. N. M. (1999). Finite size scaling in three-dimensional bootstrap percolation. Ann. Probab. 27 1837-1850. MR1742890

[5] CERF, R. and MANZO, F. (2002). The threshold regime of finite volume bootstrap percolation. Stochastic Process. Appl. 101 69-82. MR1921442

[6] CoRnell MATh EXPlorers' Club. Available at http://www.math.cornell.edu/ mec/ 2003-2004/graphtheory/slither/howtoplayslither.html.

[7] Fraenkel, A. S., Scheinerman, E. R. and Ullman, D. (1993). Undirected edge geography. Theoret. Comput. Sci. 112 371-381. MR1216328

[8] Froböse, K. (1989). Finite-size effects in a cellular automaton for diffusion. J. Stat. Phys. 55 1285-1292. MR1002492

[9] GARDNer, M. (1972). Mathematical games. Sci. Am. 227 176-182.

[10] Holroyd, A. E. (2003). Sharp metastability threshold for two-dimensional bootstrap percolation. Probab. Theory Related Fields 125 195-224. MR1961342

[11] Holroyd, A. E. (2006). The metastability threshold for modified bootstrap percolation in $d$ dimensions. Electron. J. Probab. 11 418-433 (electronic). MR2223042

[12] Holroyd, A. E., Marcovici, I. and Martin, J. B. (2015). Percolation games, probabilistic cellular automata, and the hardcore model. Preprint. Available at arXiv:1503.05614.

[13] HOLROYD, A. E. and MARTIN, J. B. Galton-Watson games. In preparation.

[14] Hopcroft, J. E. and KARP, R. M. (1973). An $n^{5 / 2}$ algorithm for maximum matchings in bipartite graphs. SIAM J. Comput. 2 225-231. MR0337699

[15] Lichtenstein, D. and SipSER, M. (1980). GO is polynomial-space hard. J. ACM $27393-$ 401. MR0567056

[16] Renault, G. and Schmidt, S. (2015). On the complexity of the misère version of three games played on graphs. Theoret. Comput. Sci. 595 159-167. MR3369961

[17] SCHAEFER, T. J. (1978). On the complexity of some two-person perfect-information games. J. Comput. System Sci. 16 185-225. MR0490917

[18] SchonmanN, R. H. (1992). On the behavior of some cellular automata related to bootstrap percolation. Ann. Probab. 20 174-193. MR1143417 
[19] Spencer, J. (2001). The Strange Logic of Random Graphs. Algorithms and Combinatorics 22. Springer, Berlin. MR1847951

[20] Wästlund, J. (2012). Replica symmetry of the minimum matching. Ann. of Math. (2) 175 1061-1091. MR2912702

R. BASU

DEPARTMENT OF MATHEMATICS

STANFORD UNIVERSITY

STANFORD, CALIFORNIA 94305-2125

USA

E-MAIL: rbasu@stanford.edu

\section{J. B. MARTIN}

STATISTICS DEPARTMENT

UNIVERSITY OF OXFORD

OXFORD OX1 3LB

UNITED KINGDOM

E-MAIL:martin@stats.ox.ac.uk

\author{
A. E. Holroyd \\ MicRosoft RESEARCH \\ REDMOND, WASHINGTON \\ USA \\ E-MAIL: holroyd@microsoft.com \\ J. WÄSTLUND \\ Department of Mathematical SCIENCES \\ Chalmers University of TECHNOLOGY \\ SE-412 96, GÖTEBORG \\ SWEDEN \\ E-MAIL: wastlund@chalmers.se
}

